Pacific Journal of Mathematics

MATRICES OF QUATERNIONS 


\title{
MATRICES OF QUATERNIONS
}

\author{
J. L. BRENNER
}

1. Introduction. In this note, some theorems which concern matrices of complex numbers are generalized to matrices over real quaternions. First it is proved that every matrix of quaternions has a characteristic root. Next, there exist $n-1$ mutually orthogonal unit $n$-vectors all orthogonal to a given vector. It is shown that Schur's lemma holds for matrices of quarternions: every matrix can be transformed into triangular form by a unitary matrix. For individual quaternions, it is known that two quaternions are similar if they have the same trace and the same norm-thus every quaternion has a conjugate $a+b j(b \geq 0)$. This fact is proved again.

The quaternion $\lambda$ is called a characteristic root of a (square) matrix $A$ provided a non-zero vector $x$ exists such that $A x=x \lambda$. Similar matrices have the same characteristic roots; if $y=T x$, where $T$ has an inverse, then $T A T^{-1} y$ $=T A x=T x \lambda=y \lambda$. Another interesting fact is that if $\lambda$ is a characteristic root, then so is $\rho^{-1} \lambda \rho$; for from $A x=x \lambda$ follows $A(x \rho)=(x \rho) \rho^{-1} \lambda \rho$; thus if the vector corresponding to the characteristic root $\lambda$ is $x$, then $x \rho$ is the vector corresponding to the characteristic root $\rho^{-1} \lambda \rho$.

2. Lemma. We shall need the following result.

Lemma 1. If $A=\left(a_{i, j}\right)$ is a matrix of elements from any field or fields, then a triangular matrix $T$ exists such that $T^{-1} A T=C=\left(c_{i, j}\right)$, where $c_{i, j}=0$ whenever $i>j+1$. The elements of $T$ are rational functions of the elements of $A$.

Proof. The proof consists in transforming $A$ in steps so that an additional zero appears at each step. First $A$ is transformed so that all the elements in the first column (except the first two) become zero; the transformed matrix is further transformed so that all the elements in the second column (except the first three) become zero, and so on. The formal proof is inductive; it will be sufficient to give the idea of the proof. In the first column of $A$, either $a_{j, 1}=0$ for all $j>1$, or else 
$a_{j, 1} \neq 0$ for some $j>1$. In the former case, proceed directly to the second column. In the other case, assume without loss of generality that $a_{2,1} \neq 0$ (otherwise transform by a permutation matrix). Let $I$ stand for the identity matrix, and let $e_{m, n}$ be the matrix with 1 in the $(m, n)$ th place and 0 elsewhere. Let $w_{3,2}$ be an element of the field. The transform $B=\left(b_{i, j}\right)$ of $A$ by the matrix $I+w_{3,2} e_{3,2}$ satisfies the conditions

$$
b_{2,1}=a_{2,1}, \quad b_{3,1}=a_{3,1}+w_{3,2} a_{2,1} .
$$

It is evident that if $w_{3,2}$ is suitably chosen, then the condition $b_{3,1}=0$ will be satisfied. Further transformations by

$$
l+w_{J, 2} e_{J, 2} \quad(\jmath=4, \cdots, n)
$$

will successively replace the elements in the first column of $A$ (except the first two) by zeros. The second and later columns are handled in order by the same method.

The above lemma and proof follow the lines of Lenıma 4.4 of [1]; in that reference, the elements of the matrix $A$ are residue classes mod $p^{r}$, a prime power.

3. The existence of characteristic roots. We shall show that every matrix $A$ of quaternions has a characteristic root.

Since any characteristic root of $C$ is also a characteristic root of $A$, it is enough to prove that $C$ has a characteristic root. The proof is by induction on $n$. There are two cases. First, suppose that $c_{j+1, j}=0$ for some $j$ with $j<n$. Let $C_{(j)}$ be the principal $j$-rowed minor of $C$; a non-zero vector $x_{(j)}$ and a characteristic root $\lambda$ exist such that $C_{(j)} x_{(j)}=x_{(j)} \lambda$. Then $\lambda$ is a characteristic root of $C$ : the corresponding vector is obtained from the vector $x_{(j)}$ by appending $n-j$ zeros.

In the second case, it is true for each $j$ that $c_{j+1, j} \neq 0$. There is a characteristic vector $\left(x_{1}, x_{2}, \cdots, x_{n}\right)$ with $x_{n}=1$; it is found by solving a polynomial equation of degree $n$ with just one term of highest degree. The fact that every such equation has a solution is proved in [5]. The equation in question comes by eliminating $x_{n-2}, x_{n-3}, \cdots, x_{1}$ in turn from the set $C x=x \lambda$. This set is indeed the following:

$$
\begin{gathered}
c_{n, n-1} x_{n-1}+c_{n, n}=\lambda, \\
c_{n-1, n-2} x_{n-2}+c_{n-1, n-1} x_{n-1}+c_{n-1, n}=x_{n-1} \lambda,
\end{gathered}
$$




$$
c_{2,1} x_{1}+c_{2,2} x_{2}+\cdots+c_{2, n-1} x_{n-1}+c_{2, n}=x_{2} \lambda
$$

$(n)$

$$
c_{1,1} x_{1}+c_{1,2} x_{2}+\cdots+c_{1, n-1} x_{n-1}+c_{1, n}=x_{1} \lambda \text {. }
$$

First, $\lambda$ must be eliminated from (2), (3), $\cdots(n)$, using (1). Call the resulting set $\left(2^{\prime}\right),\left(3^{\prime}\right), \cdots,\left(n^{\prime}\right)$. Then $\left(2^{\prime}\right)$ must be solved for $x_{n-2}$; the resulting expression is substituted into $\left(3^{\prime}\right)$. Next the equation so obtained is solved for $x_{n-3}$; the resulting expression is substituted into $\left(4^{\prime}\right)$, and so on. Since $c_{j+1, j}$ $\neq 0$ for each $j$, these steps are meaningful. At the last stage, $\left(n^{\prime}\right)$ becomes an equation of degree $n$ in the one unknown $x_{n-1}$. The single term of highest degree is not zero. After $x_{n-1}$ is determined, the values of $x_{n-2}, \cdots, x_{1}$ are determined from $\left(2^{\prime}\right) \cdots\left(n^{\prime}\right)$, and the value of $\lambda$ is determined from (1). The se values satisfy all requirements. This proves the following result.

THEOREM 1. Every inatrix of quaternions has a characteristic root.

For an application, we note that the $2 \times 2$ matrix $\left(a_{i, j}\right)$ has characteristic root $a_{1,1}$ corresponding to the vector $(1,0)$ if $a_{2,1}=0$. If $a_{2,1} \neq 0$, a characteristic vector is $\left(x_{1}, 1\right)$, and the corresponding characteristic root is $\lambda=a_{2,1} x_{1}+a_{2,2}$, where $x_{1}$ is a solution of $x_{1} a_{2,1} x_{1}=a_{1,1} x_{1}-x_{1} a_{2,2}+a_{1,2}$.

4. Generalization of Schur's lemma. To continue the discussion, we need:

Lemina 2. There exists a unitary ${ }^{1}$ matrix $U$ of quaternion elements which has a preassigned unit ${ }^{1}$ vector $u_{1}=\left(u_{1,1}, u_{1,2}, \cdots, u_{1, n}\right)$ in the first row.

Proof. Since the space of $n$-tuples over quaternions has the same dimension independent of the choice of basis [6, pp. 18-19], there is a set of $n$ vectors $u_{1}, b_{2}, \cdots, b_{n}$ which are linearly independent and span the space. From these an orthonormal set $u_{1}, \cdots, u_{n}$ can be constructed by Schmidt's process of orthogonalization. The matrix which has these vectors for rows is unitary. The process is exhibited in $[3, \mathrm{p.} 10]$, where, however, the first displayed equation should be changed to read $b_{k} \cdot b_{m}=\left(b_{k} \cdot a_{m}-b_{k} \cdot a_{m}\right)\|c\|^{-1}=0$; otherwise the reference $[3, p .21$, line 2$]$ to this equation would be inappropriate.

THEOREM 2. (Generalization of Schur's lemma.) Every matrix of real quaternions can be transformed into triangular form by a unitary matrix.

\footnotetext{
${ }^{1}$ A matrix $U$ is called unitary if $U U^{*}=1$. A vector $(u)$ is called a unit vector if $u u^{*}=1$.
} 
Proof. This theorem is a direct consequence of Theorem 1 and Lemma 2. The proof, given in [9, pp. 25-26], applies with equal force when the elements of the matrices are quaternions.

5. Transformations of matrices. We shall establish several lemmas.

Lemma 3. Let $q$ be a quaternion. There exists another quaternion s such that $|s|=1, s^{-1} q s=A+B j$, where $A+B j$ is a complex number with $B \geq 0$.

Lemma 3 is a consequence of Lemmas $4,5,6,7$. It is proved also in [4], which refers to [2]. Another proof is given here because this proof is so direct, and because Lemma 5 appears to be new.

Lemma 4. Let $q=A+B j+C k+D j k, s=E+F j+G k+I l j k,|s|=1$. The four components of $s^{-1}$ qs are respectively

$$
\begin{aligned}
& A, \\
& B\left[E^{2}+F^{2}-G^{2}-H^{2}\right]+2 C[F G+E H]+2 D[F H-E G], \\
& 2 B[F G-E H]+C\left[E^{2}+G^{2}-F^{2}-H^{2}\right]+2 D[E F+G H], \\
& 2 B[E G+F H]+2 C[G H-E F]+D\left[E^{2}+H^{2}-F^{2}-G^{2}\right] .
\end{aligned}
$$

Lemma 5. If $q=A+B j+C k+D j k$, then $s=E+F j$ exists such that $|s|$ $=1, s^{-1} q s$ has fourth component zero.

Proof. If $D=0$, take $s=1$. If $D \neq 0$, set $s=t /|t|$, where

$$
t=C-\left(C^{2}+D^{2}\right)^{1 / 2}+D j \text {. }
$$

Lemma 6. If $q=A+B j+C k$, then s exists such that $|s|=1 ; s^{-1}$ qs has third and fourth components both zero.

Proof. If $C=0$, take $s=1$. If $C \neq 0$, set $J=B / C$, and take $s=t /|t|$, where $t=-1+\left[J+\left(J^{2}+1\right)^{1 / 2}\right] j+k+\left[J-\left(J^{2}+1\right)^{1 / 2}\right] j k$.

Lemma 7. If $q=A+B j$, then $s$ exists such that $|s|=1 ; s^{-1} q s=A-B j$.

Proof. Take $s=(j+j k) / \sqrt{2}$.

COROLLARY. Every quaternion is similar to its conjugate.

The referee outlined another proof for the fact that two quaternions with equal 
norms and traces can be transformed one into the other. Let the quaternions be

$$
r=a_{0}+a_{1} j+a_{2} k+a_{3} j k, q=b_{0}+b_{1} j+b_{2} k+b_{3} j k .
$$

Consider then the equation $x r=q x$, where $x=x_{0}+x_{1} j+x_{2} k+x_{3} j k$. The four linear homogeneous equations for $x_{0}, x_{1}, x_{2}, x_{3}$ which are equivalent with this have as determinant an expression which under the assumption $a_{0}=b_{0}$ reduces to

$$
\left(a_{1}^{2}+a_{2}^{2}+a_{3}^{2}-b_{1}^{2}-b_{2}^{2}-b_{3}^{2}\right)^{2},
$$

which is equal to 0 under the assumptions made.

6. On characteristic roots. It has already been proved that any quaternion matrix $A$ can be transformed into triangular form $T$ by some unitary matrix. It follows further from Lemma 3 that $A$ can be transformed by a unitary matrix into triangular form in such a way that the diagonal elements are all of the form $A+B j$, $B \geq 0$. Indeed this transformation can be brought about by transforming $T$ by an appropriate unitary diagonal matrix.

The diagonal elements $A+B j(B \geq 0)$ which appear in this last transform of $A$ are unique; that is, any other transform of $A$ which is in triangular form and which has numbers $A+B j(B \geq 0)$ on the main diagonal will have the same numbers, although not necessarily in the same order.

The above fact is a consequence of general theorems concerning characteristic roots of a matrix.

THEQREM 10. If $\lambda$ is a characteristic raot of $A$, then so is $\rho \lambda \rho^{-1}$ (see page $329)$.

THEOREM 1l. If $A$ is in triangular form, then every diagonal element is a characteristic root.

Proof. Let $A=\left(a_{r, s}\right)$ be given: $a_{r, s}=0$ when $s<r$. It is trivial that $a_{1,1}$ is a characteristic root. Suppose it has been proved that $a_{1,1}, a_{2,2}, \cdots, a_{t, t}$ are characteristic roots. If $a_{t+1, t+1}$ is similar to any one of these, then $a_{t+1, t+1}$ is a characteristic root in virtue of that fact alone. If $a_{t+1, t+1}$ is similar to none of the preceding diagonal elements, then the vector $\left(x_{1}, x_{2}, \cdots, x_{t-1}, x_{t}, 1,0,0\right.$, $\cdots, 0)$ is a characteristic vector corresponding to the characteristic root $a_{t+1, t+1}$ provided all the following equations are satisfied: 


$$
\begin{aligned}
a_{t, t} x_{t}+a_{t, t+1} & =x_{t} a_{t+1, t+1}, \\
a_{t-1, t-1} x_{t-1}+a_{t-1, t} x_{t}+a_{t-1, t+1} & =x_{t-1} a_{t+1, t+1}, \\
\text {. . . . } & =x_{1} a_{t+1, t+1} .
\end{aligned}
$$

Equations of the above type have been considered in [7]. It is shown there that if $a, b, c$ are quaternions, and if $a$ is not similar to $c$, then $a x+b=x c$ has a solution. Hence the above equations can be solved in serial order.

THEOREM 12. Let a matrix of quaternions be in triangular form. Then the only characteristic roots are the diagonal elements (and the numbers similar to them).

Proof. If for some $\lambda$, we have $A x=x \lambda, x$ a non-zero vector, and if $A$ is triangular, then

$$
\begin{gathered}
a_{n, n} x_{n}=x_{n} \lambda, \\
a_{n-1, n-1} x_{n-1}+a_{n-1, n} x_{n}=x_{n-1} \lambda,
\end{gathered}
$$

If $x_{n} \neq 0$, then $\lambda$ is similar to $a_{n, n}$. If

$$
x_{n}=x_{n-1}=\cdots=x_{t+1}=0, \quad x_{t} \neq 0
$$

then $\lambda$ is similar to $a_{t, t}$.

THEOREM 13. Similar matrices have the same characteristic roots (see page 329).

The determinant-like function $\nabla$ of the matrix $A$, defined by Study in [10], is the product of the norms of the characteristic roots of $A$.

COROLLARY. The product of the norms of the characteristic roots of a matrix of quaternions is a rational integral function of the elements and their conjugates.

After this article was submitted for publication, the author learned of an article by H.C.Lee [8] which contains many of our results. The methods of proof there are different from ours. 


\section{REFERENCES}

1. J. L. Brenner, The linear homogeneous group, Ann. of Math. (2) 39 (1938), 472-493.

2. A. Cayley, On the quaternion equation $q Q-Q q^{\prime}=0$, Mess. of Math. 14 (1885), 108-112.

3. C. Chevalley, Theory of Lie groups, Princeton University Press, Princeton, 1946.

4. H. S. M. Coxeter, Quaternions and reflections, Amer. Math. Monthly 53 (1946), 137-138.

5. S. Eilenberg and I. Niven, The "fundamental theorem of algebra" for quaternions, Bull. Amer. Math. Soc. 50 (1944), 244-248.

6. N. Jacobson, Theory of rings, American Mathematical Society, New York, 1943.

7. R. E. Johnson, On the equation $X \alpha=\gamma X+\beta$ over an algebraic division ring, Bull. Amer. Math. Soc. 50 (1944), 202-207.

8. H.C. Lee, Eigenvalues and canonical forms of matrices with quaternion coefficients, Proc. Roy. Irish Acad. Sect. A, 52 (1949), 253-260.

9. F. D. Murnaghan, The theory of group representations, Johns Hopkins Press, Baltimore, 1938.

10. E. Study, Zur Theorie der linearen Gleichungen, Acta Math. 42 (1920), 1-61.

State College of Washington 



\title{
PACIFIC JOURNAL OF MATHEMATICS
}

\section{EDITORS}

\author{
HERBERT BUSEMANN \\ University of Southern California \\ R. M. RoBINSON \\ Los Angeles 7, California \\ University of California \\ Berkeley 4, California \\ E. F. BECKENBACH, Managing Editor \\ University of California \\ Los Angeles 24, California
}

\section{ASSOCIATE EDITORS}

R. P. DILWORTH
HERBERT FEDERER
MARSHALL HALL

\author{
P. R. HALMOS \\ HEINZ HOPF \\ R. D. JAMES
}

\author{
B $\emptyset$ RGE JESSEN \\ PAUL LÉVY \\ GEORGE PÓLYA
}

\author{
J. J. STOKER \\ E. G. STRAUS \\ KÔSAKU YOSIDA
}

\section{SPONSORS}

UNIVERSITY OF BRITISH COLUMBIA

CALIFORNIA INSTITUTE OF TECHNOLOGY

UNIVERSITY OF CALIFORNIA, BERKELEY

UNIVERSITY OF CALIFORNIA, DAVIS

UNIVERSITY OF CALIFORNIA, LOS ANGELES

UNIVERSITY OF CALIFORNIA, SANTA BARBARA

OREGON STATE COLLEGE

UNIVERSITY OF OREGON

\author{
UNIVERSITY OF SOUTHERN CALIFORNIA \\ STANFORD UNIVERSITY \\ WASHINGTON STATE COLLEGE \\ UNIVERSITY OF WASHINGTON

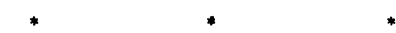 \\ AMERICAN MATHEMATICAL SOCIETY \\ NATIONAL BUREAU OF STANDARDS, \\ INSTITUTE FOR NUMERICAL ANALYSIS
}

Mathematical papers intended for publication in the Pacific Journal of Mathematics should be typewritten (double spaced), and the author should keep a complete copy. Manuscripts may be sent to any of the editors. All other communications to the editors should be addressed to the managing editor, E. F. Beckenbach, at the address given above.

Authors are entitled to receive 100 free reprints of their published papers and may obtain additional copies at cost.

The Pacific Journal of Mathematics is published quarterly, in March, June, September, and December. The price per volume (4 numbers) is $\$ 8.00$; single issues, $\$ 2.50$. Spécial price to individual faculty members of supporting institutions and to members of the American Mathematical Society: $\$ 4.00$ per volume; single issues, $\$ 1.25$.

Subscriptions, orders for back numbers, and changes of address should be sent to the publishers, University of California Press, Berkeley 4, California.

UNIVERSTTY OF CALIFORNIA PRESS • BERKELEY AND LOS ANGELES 


\section{Pacific Journal of Mathematics}

\section{Vol. 1, No. $3 \quad$ BadMonth, 1951}

R. P. Boas, Completeness of sets of translated cosines ............. 321

J. L. Brenner, Matrices of quaternions . ..................... 329

Edmond Darrell Cashwell, The asymptotic solutions of an ordinary differential equation in which the coefficient of the parameter is

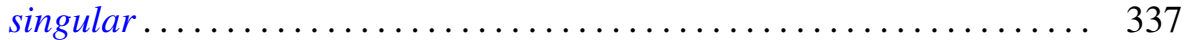

James Dugundji, An extension of Tietze's theorem ................ 353

John G. Herriot, The polarization of a lens ...................... 369

J. D. Hill, The Borel property of summability methods ............... 399

G. G. Lorentz, On the theory of spaces $\Lambda \ldots \ldots \ldots \ldots \ldots \ldots \ldots \ldots \ldots . \ldots \ldots$

J. H. Roberts and W. R. Mann, On a certain nonlinear integral equation of the Volterra type ................................. 431

W. R. Utz, A note on unrestricted regular transformations . . .......... 447

Stanley Simon Walters, Remarks on the space $H^{p} \ldots \ldots \ldots \ldots \ldots \ldots . . \ldots 55$

Hsien Chung Wang, Two theorems on metric spaces ............... 473 